\title{
Size-Dependent Structural and Magnetic Properties of Disordered Co2FeAl Heusler Alloy Nanoparticles
}

\author{
Aquil Ahmad, Srimanta Mitra, S. K. Srivastava and A. K. Das ${ }^{\dagger}$ \\ Department of Physics, Indian Institute of Technology Kharagpur, India-721302. \\ $†$ Corresponding author: amal@ phy.iitkgp.ac.in
}

\begin{abstract}
$\mathrm{Co}_{2} \mathrm{FeAl}$ (CFA) nanoparticles (NPs) of different sizes were synthesized by chemical route. The effect of the size of NPs upon the structure and magnetization compared to its bulk counterpart was investigated. The structure and composition were determined from X-ray diffraction (XRD) and electron microscopy. XRD analysis shows that the samples are having single (A2-type) disordered phase. Magnetization measurements suggest that the samples are soft ferromagnetic in nature with very low coercivity. Enhanced magnetic properties like saturation magnetization, coercive force, retentivity, and Curie-temperature are observed with a decrease in particle size. The effect of particle size on hysteresis losses is also discussed. The smallest particles of size 16 $\mathrm{nm}$ exhibited the highest saturation magnetization and transition temperature of $180.73 \mathrm{emu} / \mathrm{g}$ and $1261 \mathrm{~K}$, respectively. The origin of enhancement in magnetization of $\mathrm{Co}_{2} \mathrm{Fe} A l$ nano-alloy is attributed to the strong Co-Co exchange interaction due to disorder present in the systems.
\end{abstract}

Keywords: Heusler alloy, Saturation magnetization, Curie-Weiss temperature, Slater-Pauling rule, Half-metallicity.

\section{Introduction}

Heusler Alloys (HAs) referred to ternary intermetallic compounds having general formula $\mathrm{X}_{2} \mathrm{YZ}$ are generally called full-Heusler alloys. HAs crystallize in the cubic symmetry under space group $\mathrm{Fm} \overline{3} \mathrm{~m}$ and fully ordered in ideal L21 phase [1-3]. Full-Heusler alloys may also crystallize in disordered phases like B2 (partially disordered), A2 (fully disordered) and DO3 type phases [4-6]. These alloys show multifunctional properties, like half-metallic ferromagnetism (HMF), shape-memory effect, magneto-caloric effect, and thermo-electric behavior [7-10]. Band-structure calculations divulge that these compounds are half-metallic ferromagnets (HMF), i.e., 100\% spin-polarized near Fermi level. Over the past few years, Heusler compounds are considered as the most promising class of materials for spintronics and magneto-electronics applications due to the theoretical prediction of their half-metallicity, high Curie-Weiss temperature $\left(\mathrm{T}_{\mathrm{c}}\right)$ and structural similarity with the binary semiconductors. However, in some alloys, less spin polarization is observed [11,12] due to the structural disorder $[13,14]$ and surface effect [15]. Heusler nanoparticles (NPs) exhibit all the bulk features with different magnitudes and hence it is worth to study the crucial factors, like how size and shape of the nanoparticles affect the structural and magnetic properties of Heusler nanoparticles.

Among all known Heusler alloys, $\mathrm{Co}_{2}$ based Heusler alloys, particularly; $\mathrm{Co}_{2} \mathrm{FeAl}$ (CFA) attracts more attention due to its unique properties, like high saturation magnetization $\left(\mathrm{M}_{\mathrm{s}}\right)$ and high Curie-Weiss temperature $\left(\mathrm{T}_{\mathrm{c}}\right)$. The experimental lattice parameter of bulk CFA was found 
to be $5.73 \AA$ and saturation magnetization was obtained around $5.20 \mu_{\mathrm{B}} / \mathrm{f}$.u. at room temperature (RT) [16]. Theoretically, the calculated spin magnetic moment was found to be $4.89 \mu_{\mathrm{B}} / \mathrm{f}$.u. using experimental lattice constant, this theoretical value was slightly less than the predicted value of CFA by the Slater-Pauling (SP) rule, which was $5.0 \mu_{\mathrm{B}} / \mathrm{f}$.u. [10]. The $\mathrm{T}_{\mathrm{c}}$ of bulk CFA was predicted $\geq 1100 \mathrm{~K}$ [17]. In recent years, CFA has been investigated extensively, but studies are limited to bulk and thin-films only due to very complicated process of nano-particle synthesis. Phase separation can easily occur in the case of ternary intermetallic alloys due to immiscibility and lattice mismatch; so no particular methodology of synthesis process has been developed till date to achieve nominal phase and stoichiometry. Heusler NPs are also the material of interest as their structural and magnetic properties can easily be tuned by controlling the shape and size of the particles; hence, they are having great importance in the fabrication of nano-devices in spintronics, drug delivery, catalysis and biomedical imaging applications like other magnetic nanostructures mostly based on binary alloys [18-23]. There are very few reports on CFA nanoparticles (NPs); however, the size-dependent studies on $\mathrm{Co}_{2} \mathrm{FeAl}$ NPs are not done yet.

Nanostructured CFA was prepared by a hydrothermal method using Teflon-autoclave by Fujun Yang et al. [24]. They have shown the dependency of saturation magnetization $\left(\mathrm{M}_{\mathrm{s}}\right)$ and coercivity $\left(\mathrm{H}_{\mathrm{c}}\right)$ on the reaction time and obtained a maximum saturation magnetization of 189.2 emu/g. Keshab R et al. [25] also prepared CFA nanowires of diameter ranging from 50 to 500 $\mathrm{nm}$ with a lattice constant of $5.639 \AA$. They found that the lattice constant of prepared nanowires was within $1 \%$ of the lattice parameter of the bulk system. Hollow CFA NPs were also prepared by using capping agent, namely polyethylene glycol polymer and then annealed at $700{ }^{\circ} \mathrm{C}$ with a heating range from 5 to $15^{\circ} \mathrm{C}$ per min [26]. They achieved a maximum saturation magnetization of $95 \mathrm{emu} / \mathrm{g}$ and $\mathrm{H}_{\mathrm{c}}$ of $730 \mathrm{Oe}$. Al Kanani et al. found Tc above $1000 \mathrm{~K}$ for bulk CFA alloy and the saturation magnetization of $5.1 \mu_{\mathrm{B}} / \mathrm{f}$.u. [27]. A. Kumar also chemically prepared CFA nanoparticles in size range of 10-50 $\mathrm{nm}$ and reported that nanoparticles were having disordered mixed phases of B2 and A2 types [28]. J. H. Du et al. prepared CFA nanoparticles using coprecipitation method followed by $\mathrm{H}_{2}$ annealing and obtained particles in the size range of 50-400 $\mathrm{nm}$ with $\mathrm{M}_{\mathrm{S}}=139 \mathrm{emu} / \mathrm{g}$ at $300 \mathrm{~K}$ [29]. Lana T. Huynh et al. prepared off-stoichiometric CFA nanocrystals by thermal decomposition of the corresponding metal acetylacetonate complexes in the presence of capping ligands, and then annealed in presence of $\mathrm{H}_{2}$ gas [30]. They showed that the saturation magnetization was increased with annealing temperature from $4.0 \mu_{\mathrm{B}} /$ f.u. to 5.3 $\mu_{\mathrm{B}} /$ f.u.

In this paper, we report the size effect on structural and magnetic properties of CFA NPs having a mean size of $16 \pm 10 \mathrm{~nm}$ and $190 \pm 9 \mathrm{~nm}$, respectively.

\section{Experiments}

CFA nanoparticles of average sizes of $190 \mathrm{~nm}$ and $16 \mathrm{~nm}$ within the variation of $\pm 10 \mathrm{~nm}$ were synthesized by co-precipitation and thermal deoxidization method reported by J.H. Du et al. [29] with slight modification. We used the precursor salts: $\mathrm{CoCl}_{2} \cdot 6 \mathrm{H}_{2} \mathrm{O}(99 \%), \mathrm{Fe}\left(\mathrm{NO}_{3}\right)_{3} .9 \mathrm{H}_{2} \mathrm{O}(99 \%)$ and $\mathrm{Al}_{2}\left(\mathrm{NO}_{3}\right) \cdot 18 \mathrm{H}_{2} \mathrm{O}$ (98\%). The chemicals from Sigma-Aldrich (American Chemicals Company) were used without any further purification. In a typical preparation of the sample, 0.6 mmol of $\mathrm{Al}_{2}\left(\mathrm{NO}_{3}\right) .18 \mathrm{H}_{2} \mathrm{O}, 1.2 \mathrm{mmol}$ of $\mathrm{Fe}\left(\mathrm{NO}_{3}\right)_{3} .9 \mathrm{H}_{2} \mathrm{O}$ and $2.4 \mathrm{mmol}$ of $\mathrm{CoCl}_{2} .6 \mathrm{H}_{2} \mathrm{O}$ were dissolved in $50 \mathrm{ml}$ methanol and then dried for more than 12 hours in $100{ }^{\circ} \mathrm{C}$. Obtained dried 
powder was further annealed at $800{ }^{\circ} \mathrm{C}$ for 3 hours and 12 hours in presence of $\mathrm{H}_{2}$ atmosphere to deoxidize the powder.

Phase identification was studied by high-resolution x-ray diffractometer (HR-XRD) using $\mathrm{Cu}$ $\mathrm{K} \alpha$ radiation from (i) Bruker D2 Phaser x-ray diffractometer and (ii) PANalytical's X-ray diffractometer. The morphologies of prepared NPs were analyzed by MERLIN field-emission gun scanning electron microscope (FESEM), which was operated at $200 \mathrm{kV}$. The colloidal nanoparticles were pre-sonicated in acetone, a small drop was placed in a small Si substrate and then gold coating was used to prepare the sample for FESEM, this drop was further placed on a carbon supported $\mathrm{Cu}$ (Copper) grid for the transmission electron microscope (TEM). To determine the purity and composition energy-dispersive X-ray analysis (EDAX) was used. DC magnetization measurements were carried out using a physical property measurement system (PPMS) of Cryogenic Limited, UK and high-temperature vibrating sample magnetometer (VSM) of LAKESHORE, USA.

\section{Results and discussions}

\subsection{X-ray diffraction (XRD)}

XRD patterns of all the prepared $\mathrm{Co}_{2} \mathrm{FeAl}$ (CFA) samples annealed at $800{ }^{\circ} \mathrm{C}$ for (a) 3 hours and (b) 12 hours are shown in Fig. 1. The pattern consists of three clear characteristic peaks at $44.96^{\circ}, 65.50^{\circ}$, and $82.92^{\circ}$. These peaks are indexed as (220), (400) and (422) reflections of pure $\mathrm{Co}_{2} \mathrm{FeAl}$ phase according to the Joint Committee on Powder Diffraction Standards (JCPDS) data. It is observed that the peaks are relatively displaced towards higher $2 \theta$ values compared to

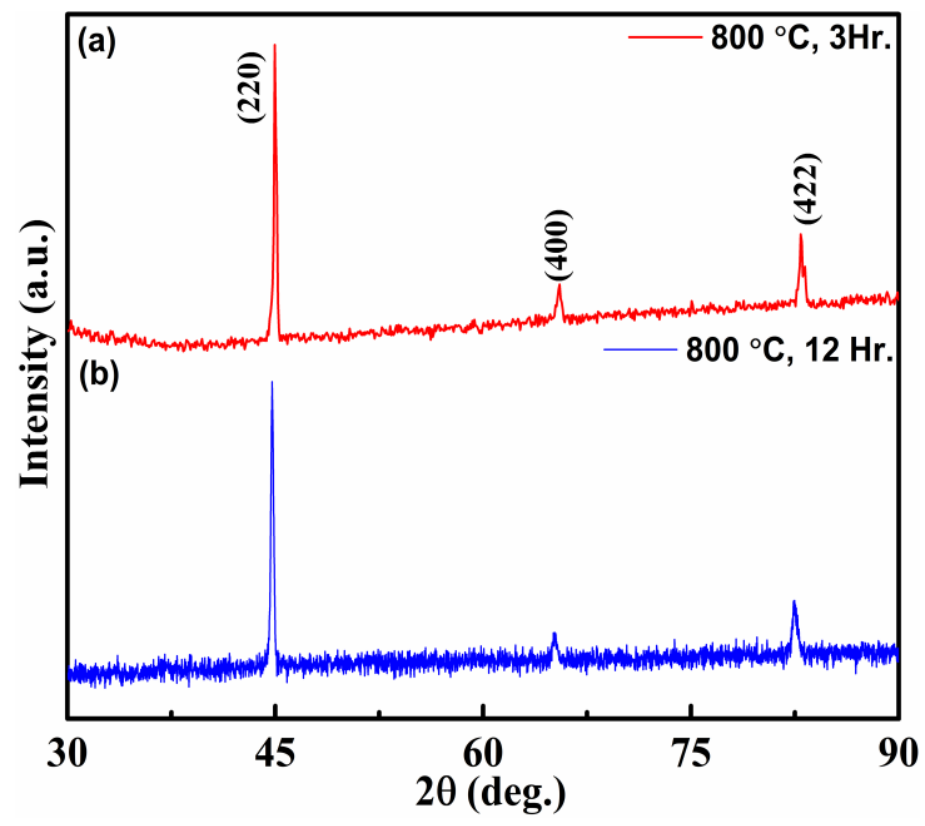

Fig. 1: XRD patterns of the $\mathrm{Co}_{2} \mathrm{FeAl}(\mathrm{CFA})$ nanoparticles annealed at $800^{\circ} \mathrm{C}$ for (a) 3 hours and (b) 12 hours.

their bulk counterpart, so the NPs are pure in phase. Furthermore, XRD patterns reveal the crystalline nature of the samples. The calculated lattice constants are of $5.695 \AA$ and $5.718 \AA$ for 
3 hours and 12 hours annealed samples, respectively, which are comparable to the bulk lattice constant [16]. From the literature, it is well known that the ideal L2 $2_{1}$ structure is recognized if (111) and (200) superlattice peaks are present in XRD patterns [31], which are not present in our XRD spectra as shown in Fig. 1. These superlattice peaks are much weaker for the elements belong to a same period in the periodic table and hence intensities of these peaks could not be easily detectable if the transition elements in the Heusler-compounds belong to the same period. These peaks are also not existed if samples have A2-type disordered structure. Subsequently, the $\mathrm{Al}$ atom in the periodic table belongs to a different period than that of $\mathrm{Co}$ or $\mathrm{Fe}$, so our samples exhibited an A2 type disordered phase [7].

\subsection{Field emission scanning electron microscopy (FESEM) and transmission electron microscopy (TEM)}

Field emission scanning electron microscopy (FESEM) images in Fig. 2 are indicating that all the particles are spherical in shape and agglomerated. The agglomeration may be due to the magnetic nature of the particles.
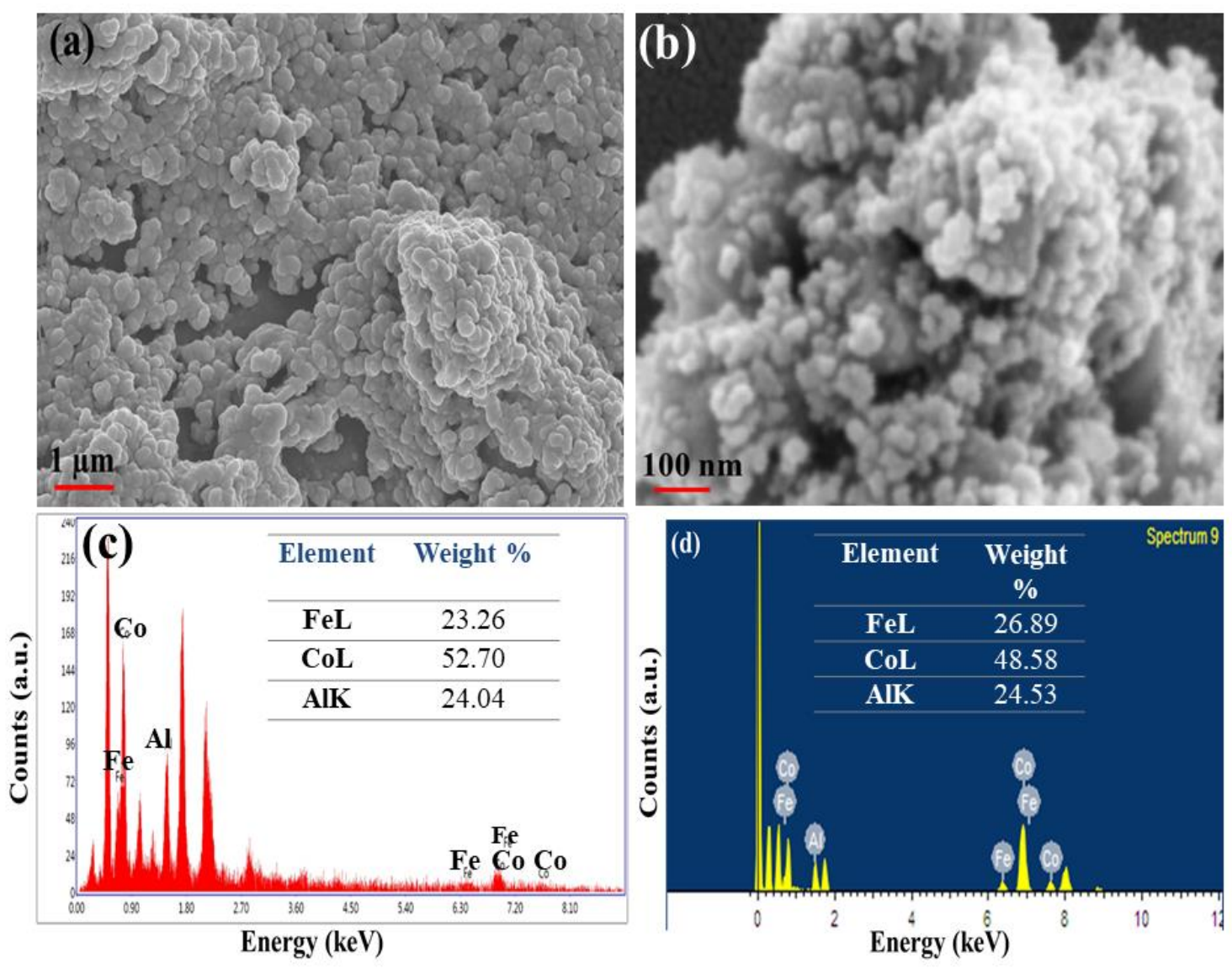

Fig. 2: FESEM images of the $\mathrm{Co}_{2} \mathrm{FeAl}$ (CFA) nanoparticles annealed at $800^{\circ} \mathrm{C}$ for (a) 3 hours and (b) 12 hours. EDAX spectrum of CFA annealed at $800^{\circ} \mathrm{C}$ for (c) 3 hours and (d) 12 hours. 
To confirm the purity and composition we have performed energy-dispersive x-ray analysis (EDAX) shown in Fig. 2 (c) and (d), which reveal that there are no foreign elements. Note that the extra peaks in the spectrum are from Si substrate and Au as the gold coating was done on the $\mathrm{Si}$ substrate during the sample preparation. This composition examination demonstrated that the deviation from the stoichiometric composition of all the samples is insignificant.

To analyze particles further we have performed transmission electron microscopy (TEM) imaging along with selected area electron diffraction (SAED) and shown in Fig. 3. The SAED patterns encompass concentric rings with some dots, which is indicating the single crystalline nature of the nanoparticles but randomly oriented in powder form. Therefore, the NPs are essentially crystalline in nature. Primary four rings of the SAED patterns are indexed with (hkl) as shown in Fig. 3 and those (hkl) values are also in good agreement with the XRD results. We analyzed all particles by using Image $J$ software and the histogram of size distribution of nanoparticles is shown in Fig. 3 and fitted with a Gaussian profile, which gives the average particle sizes of $190 \pm 9 \mathrm{~nm}$ and $16 \pm 10 \mathrm{~nm}$ for 3 hours and 12 hours annealed samples, respectively.
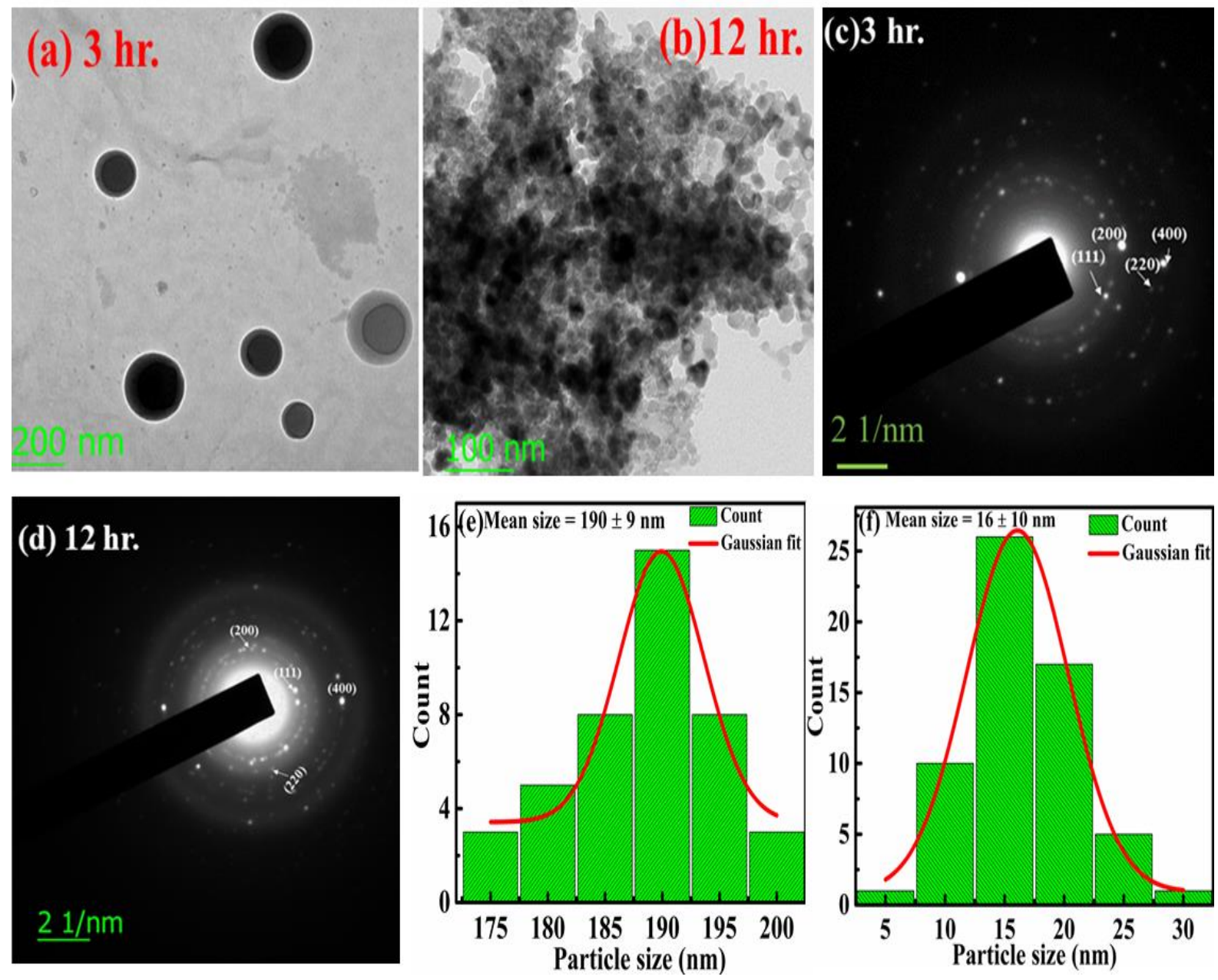
Fig. 3: TEM images of $\mathrm{Co}_{2} \mathrm{FeAl}(\mathrm{CFA}) \mathrm{NPs}$ annealed at $800{ }^{\circ} \mathrm{C}$ for (a) 3 hours and (b) 12 hours. SAED patterns of CFA annealed at $800{ }^{\circ} \mathrm{C}$ for (c) 3 hours and (d) 12 hours, with an index of initial four rings. (e) and (f) show the histogram of particle size distribution for 3 hours and 12 hours annealed samples, respectively.

\subsection{Effects of particle size on magnetic properties}

Magnetization as a function of the magnetic field was measured at low temperature $(5 \mathrm{~K})$ and at room temperature (RT). It is seen that the particles are ferromagnetic in nature and the saturation magnetization $\left(\mathrm{M}_{\mathrm{s}}\right)$ is increased with the decreasing of particle size. The $\mathrm{M}_{\mathrm{s}}$ are 147.62 $\mathrm{emu} / \mathrm{g}$ and $180.73 \mathrm{emu} / \mathrm{g}$ for $190 \mathrm{~nm}$ and $16 \mathrm{~nm}$ sized NPs, respectively. The same tendency is observed in case of coercivity $\left(\mathrm{H}_{\mathrm{c}}\right)$ and remanence $\left(\mathrm{M}_{\mathrm{r}}\right)$; both coercivity and remanence are also increased with decrease in particle size at $5 \mathrm{~K}$. The $\mathrm{H}_{\mathrm{c}}$ are $121.13 \mathrm{Oe}$ and $175.50 \mathrm{Oe}$ and the $\mathrm{M}_{\mathrm{r}}$ are $2.63 \mathrm{emu} / \mathrm{g}$ and $8.05 \mathrm{emu} / \mathrm{g}$ for $190 \mathrm{~nm}$ and $16 \mathrm{~nm}$-sized nanoparticles, respectively at $5 \mathrm{~K}$. Similar trend are also observed at room temperature. Moreover, the saturation magnetization
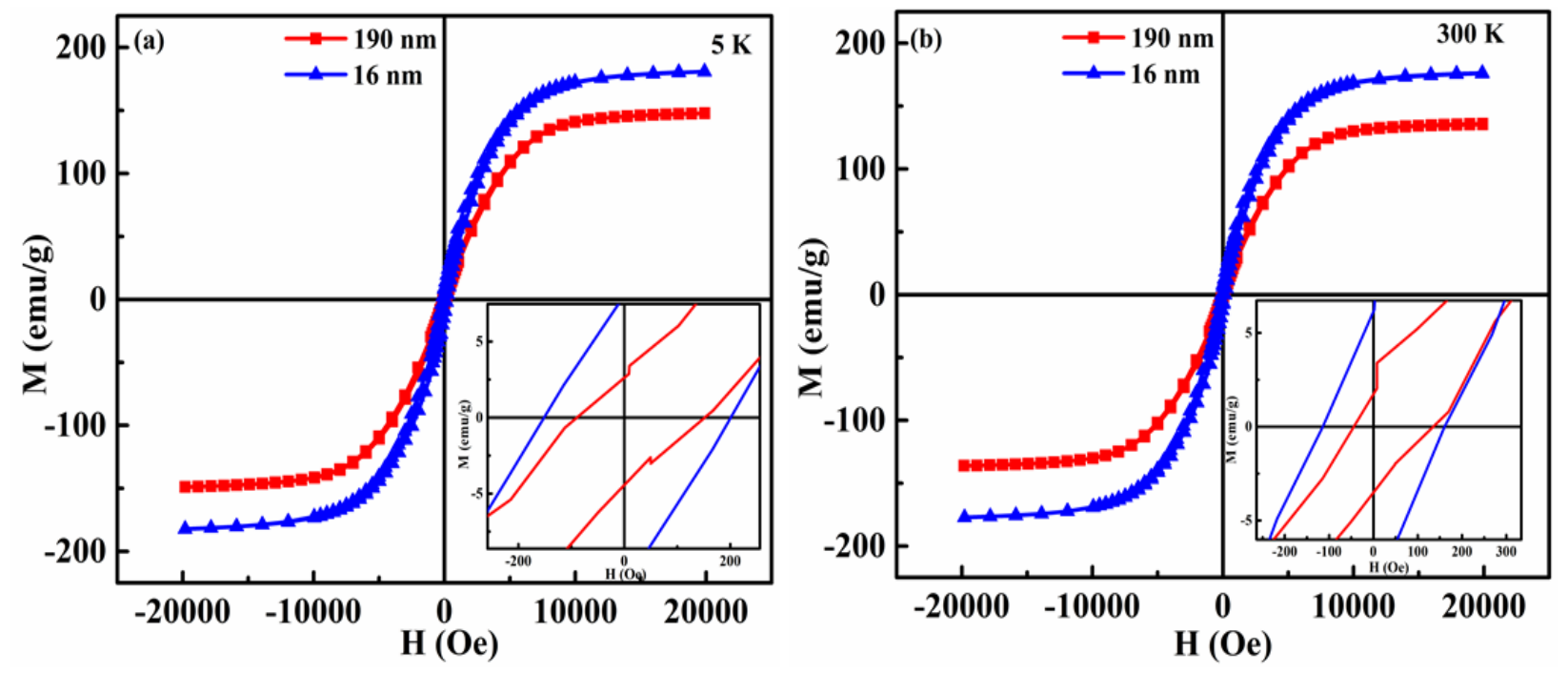

Fig. 4: Field dependence of magnetization at (a) $5 \mathrm{~K}$ and (b) $300 \mathrm{~K}$. Insets show the magnified curves around zero magnetic field.

$\left(M_{s}\right)$ is much stable even up to RT, which has great importance for device application. From Fig. 4 , it can be seen that all particles are soft ferromagnets due to very small coercivity $\left(\mathrm{H}_{\mathrm{c}}\right)$. The particles having the smallest size of $16 \mathrm{~nm}$ exhibited a highest magnetic moment of 180.73 $\mathrm{emu} / \mathrm{g}\left(\sim 6.5 \mu_{\mathrm{B}} / \mathrm{f} . \mathrm{u}\right.$.), which is also comparable with the $\mathrm{M}_{\mathrm{s}}$ value of $189.2 \mathrm{emu} / \mathrm{g}$ reported by $\mathrm{F}$. Yang et al. [24]. This value is much larger than the value reported for the bulk system (see in table 1). The enhancement in the magnetic moment and also in Curie temperature compared to bulk is attributed to the disorder (A2-type) present in our system, which leads to strong magnetic exchange interaction and as a result large magnetic moment and Curie temperature. The strong exchange interaction is basically due to the Co-Co exchange interactions. As in fully-disordered $\mathrm{CFA}$ lattice, the $\mathrm{Co}$ atom will have $\mathrm{Fe}$ or $\mathrm{Al}$ as its nearest neighbor which can lead Co-Al-Co 
exchange interaction [7]. According to Slater-Pauling (SP) rule, the theoretical bulk saturationmagnetization value for CFA is $5.0 \mu_{\mathrm{B}} / \mathrm{f}$.u. [10]. In our case, for $190 \mathrm{~nm}$-sized particles saturation magnetization value is in good agreement with the theoretical bulk value. When we go to nanoregime of particle size of $16 \mathrm{~nm}$, this value becomes $180.73 \mathrm{emu} / \mathrm{g}$, at $5 \mathrm{~K}$ and maintained even up to room temperature. A comparison of all values obtained at $5 \mathrm{~K}$ and $300 \mathrm{~K}$ has shown in Table 1. We have also measured magnetic hysteresis loops at $100 \mathrm{~K}$ and $200 \mathrm{~K}$ for $190 \mathrm{~nm}$ and $16 \mathrm{~nm}$-sized particles and the obtained values are shown in Fig. 6.

Table. 1: Saturation magnetization, remanence, coercivity, Curie temperature $\left(\mathrm{T}_{\mathrm{c}}\right)$ of $\mathrm{Co}_{2} \mathrm{FeAl}$ nanoparticles of sizes $190 \mathrm{~nm}$ and $16 \mathrm{~nm}$.

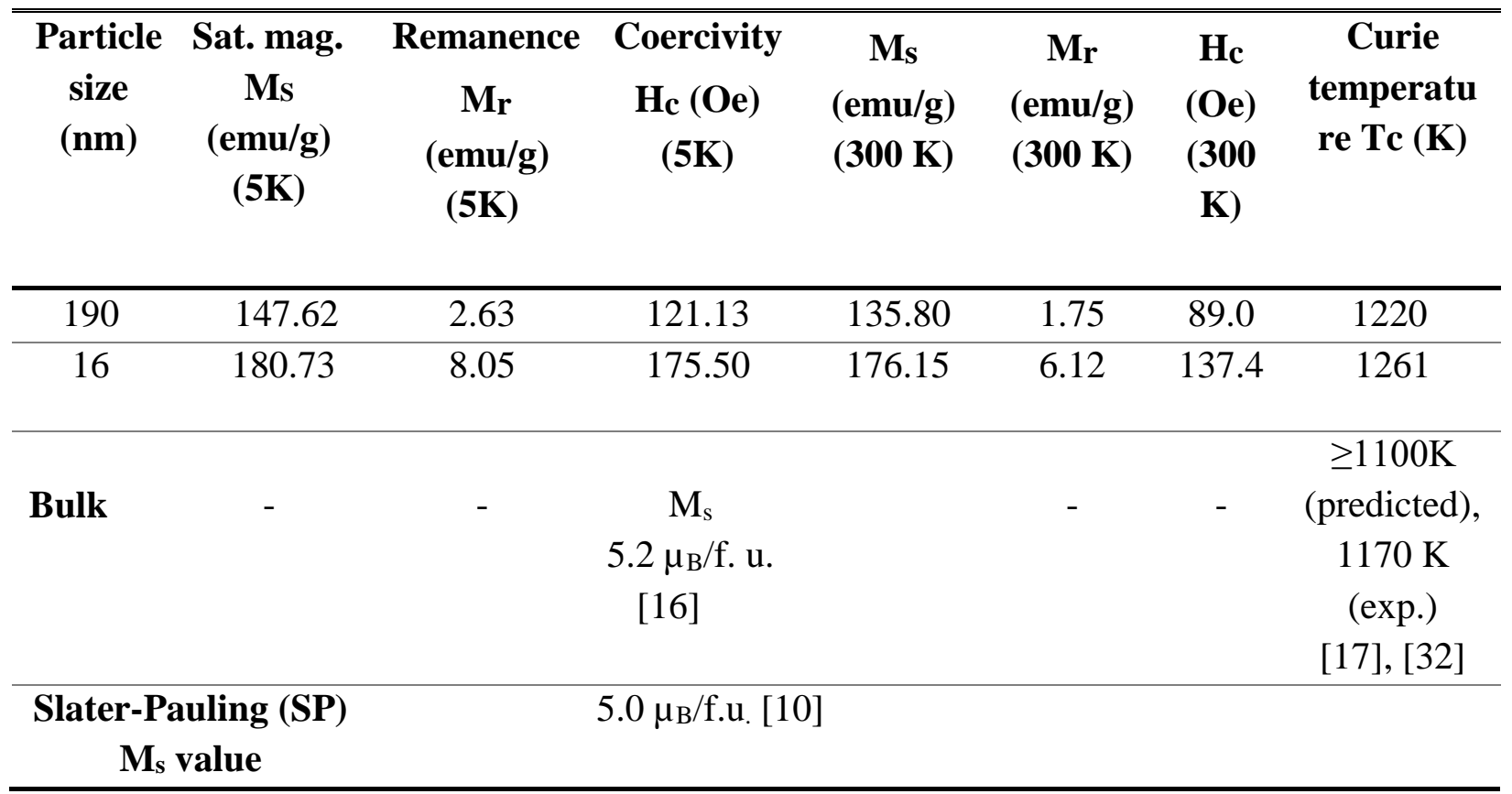

Fig. 5 shows the variation of magnetization with respect to temperature under an applied field of 100 Oe with standard ZFC (zero-field cooling) technique. The inset shows $\mathrm{dM} / \mathrm{dT}$ versus T plots for particles sized of $190 \mathrm{~nm}$ and $16 \mathrm{~nm}$. From the temperature dependence of the magnetization curve, it is clear that all samples undergo ferromagnetic (FM) to paramagnetic (PM) phase transition at Tc equals to 1220 and $1261 \mathrm{~K}$, respectively. Thus, $\mathrm{T}_{\mathrm{c}}$ increases with the decrement of particle sizes, which is very pronounced. We are not aware of any measurement of Curietemperature $\left(\mathrm{T}_{\mathrm{c}}\right)$ for CFA nanoalloy, so we could compare our experimental values with its bulk counterpart and it is much larger than its bulk value measured experimentally and predicted theoretically (see in Table.1). However, Changhai Wang et al. studied Co-Ni-Ga Heusler nanoparticles and reported highest $\mathrm{T}_{\mathrm{c}}$ of $1174 \mathrm{~K}$ for $84 \mathrm{~nm}$ sized particles [33].

The largest bulk magnetic moment and Curie temperature were predicted for $\mathrm{Co}_{2} \mathrm{FeSi}$ according to SP rule and Fecher et al. found an experimental magnetic moment and $\mathrm{T}_{\mathrm{c}}$ of 5.97 $\mu_{\mathrm{B}} /$ f.u. and $1100 \mathrm{~K}$, respectively for $\mathrm{Co}_{2} \mathrm{FeSi}$ alloy [34]. However, we found highest $\mathrm{M}_{\mathrm{s}}$ for $\mathrm{Co}_{2} \mathrm{FeAl}$ nanoparticles, which is higher than $6 \mu_{\mathrm{B}} / \mathrm{f}$.u. Our sample with mean size of $16 \mathrm{~nm}$, exhibited highest $T_{c}$ among all Heusler alloy till date. From these results, we can conclude that 
$\mathrm{Co}_{2+\mathrm{x}} \mathrm{Fe}_{1-\mathrm{x}} \mathrm{Z}$ (where $\mathrm{Z}$ is $\mathrm{Al}$ and $\mathrm{Ga}$ ) in the $\mathrm{A} 2$ phase would be the best among all Heusler alloys. It is also interesting to investigate the size effect on hysteresis losses, which is an important parameter for evaluating the performance of magnetic refrigerant materials, where minimization of hysteresis losses is better for higher refrigerant capacity (RC). Therefore, the magnetic hysteresis of ferromagnetic materials described by magnetic coercivity is worth to study.

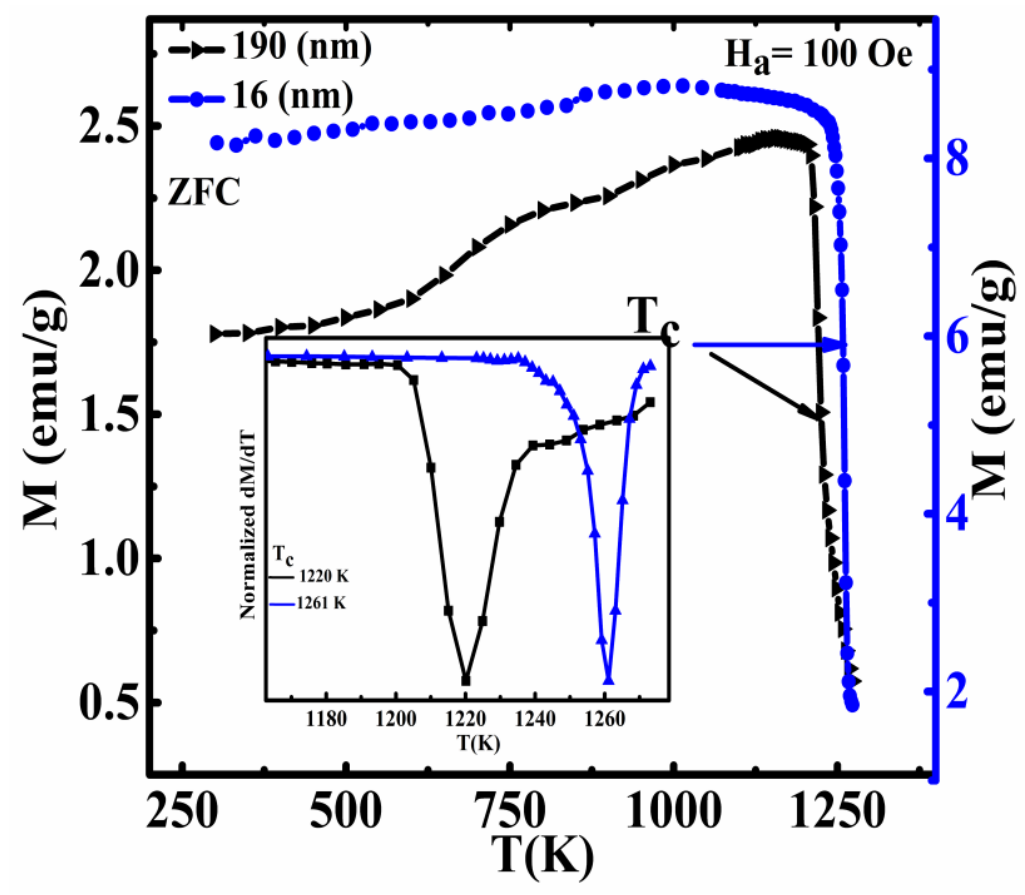

Fig. 5: Temperature dependence of magnetization, Inset shows $d M / d T$ near the transition region as a function of temperature for the particles of sizes of $190 \mathrm{~nm}$ and $16 \mathrm{~nm}$.

From Fig. 6, It has been observed that coercivity $\left(H_{c}\right)$ and remanence $\left(M_{r}\right)$ is increased as nanoparticle size goes from $190 \mathrm{~nm}$ to $16 \mathrm{~nm}$. Moreover, it is evident that at any given -

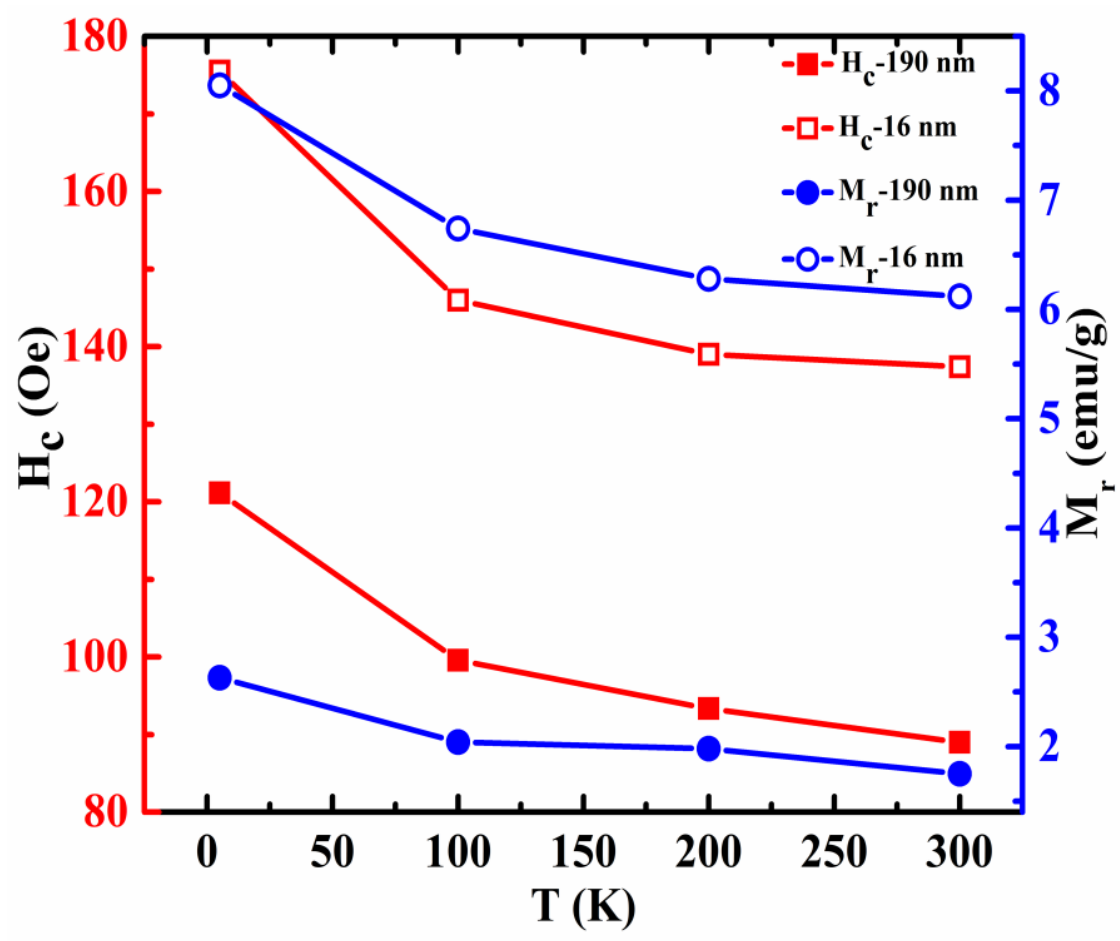


Fig. 6: (color online) Coercivity (Hc) and remanence (Mr) plot of CFA-NPs as a function of temperature for the particles sizes of $190 \mathrm{~nm}$ and $16 \mathrm{~nm}$.

temperature, magnetization, and size of the nanoparticles are inversely related and hence when the particle size is reduced, the magnetization is increased. The coercivity and remanence of the ferromagnetic NPs are strongly dependent upon the temperature. This happens because when temperature increases, more thermal energy is provided to the system. Hence, all individual electron spins will be in higher energy state, so pointing randomly with respect to neighboring spin and hence magnetization will be reduced; as a result, a small field is required to reduce the remanent magnetization to zero, therefore leading to reduction in coercivity $[35,36]$.

Here, we have shown that the magnetic moment and Curie temperature can be tuned significantly by controlling the shape and size of the nanoparticles.

\section{Summary}

In summary, we successfully synthesized different size of nearly monodispersed $\mathrm{Co}_{2} \mathrm{FeAl}$ nanoparticles and obtained the smallest mean size of $16 \mathrm{~nm}$. All samples are pure in phase and have crystallized in the A2 type of disordered phase. Our studies clearly reveal that the disorders, which are present in our CFA nanoalloys, lead to enhance saturation magnetization and Curie temperature $\left(\mathrm{T}_{\mathrm{c}}\right)$ when particle sizes are reduced. We found the maximum saturation magnetization of $180.73 \mathrm{emu} / \mathrm{g}\left(\sim 6.5 \mu_{\mathrm{B}} / \mathrm{f}\right.$.u. $)$, and Curie temperature of $1261 \mathrm{~K}$ for the particles with a mean size of $16 \mathrm{~nm}$. We found the drastic change in magnetic moment and Curie temperature $\left(\mathrm{T}_{\mathrm{c}}\right)$ with the size of the nanoparticles as compared to its bulk counterpart. The study of hysteresis losses also suggests the vitality of these low-cost materials for magnetic refrigeration technology. Our finding of CFA alloy having a high magnetic moment and Curie temperature would be interesting for fundamental science and important for device applications.

\section{Acknowledgments}

Aquil Ahmad sincerely acknowledges the University Grant Commission (UGC) Delhi, India for providing fellowship for Ph.D. work. A. K. Das acknowledges the financial support of DST, India (project no. EMR/2014/001026). We also acknowledge the Central Research Facility of IIT Kharagpur and the use of PPMS facility in the Department of Physics of IIT Kharagpur. We are also thankful to Dr. Koushik Biswas, Department of Metallurgical and Materials Engineering, IIT Kharagpur for providing $\mathrm{H}_{2}$ annealing facility.

\section{References}

[1] T. Graf, C. Felser, S.S.P. Parkin, Simple rules for the understanding of Heusler compounds, Progress in Solid State Chemistry, 39 (2011) 1-50. 
[2] T. J. Burch, T. Litrenta, J.I. Budnick, Hyperfine studies of site occupation in ternary systems, Physical Review Letters, 33 (1974) 421-424.

[3] Y. Nishino, H. Kato, M. Kato, U. Mizutani, Effect of off-stoichiometry on the transport properties of the Heusler-type $\mathrm{Fe}_{2} \mathrm{VAl}$ compound, Physical Review B, 63 (2001) 233303.

[4] T. Graf, F. Casper, J. Winterlik, B. Balke, G.H. Fecher, C. Felser, Crystal structure of new Heusler compounds, Zeitschrift für anorganische und allgemeine Chemie, 635 (2009) 976981.

[5] M.S. Gabor, M. Belmeguenai, T. Petrisor, C. Ulhaq-Bouillet, S. Colis, C. Tiusan, Correlations between structural, electronic transport, and magnetic properties of $\mathrm{Co}_{2} \mathrm{FeAl}_{0.5} \mathrm{Si}_{0.5}$ Heusler alloy epitaxial thin films, Physical Review B, 92 (2015) 054433.

[6] G. Bacon, J. Plant, Chemical ordering in Heusler alloys with the general formula $\mathrm{A}_{2} \mathrm{BC}$ or ABC, Journal of Physics F: Metal Physics, 1 (1971) 524-532.

[7] R.P. Dulal, B.R. Dahal, A. Forbes, I.L. Pegg, J. Philip, Large magnetization and high Curie temperature in highly disordered nanoscale $\mathrm{Fe}_{2} \mathrm{CrAl}$ thin films, Journal of Magnetism and Magnetic Materials, 423 (2017) 314-317.

[8] X.Y. Dong, C. Adelmann, J.Q. Xie, C.J. Palmstrøm, X. Lou, J. Strand, P.A. Crowell, J.P. Barnes, A.K. Petford-Long, Spin injection from the Heusler alloy $\mathrm{Co}_{2} \mathrm{MnGe}$ into $\mathrm{Al}_{0.1} \mathrm{Ga}_{0.9} \mathrm{As} / \mathrm{GaAs}$ heterostructures, Applied Physics Letters, 86 (2005) 102107.

[9] Y. Sakuraba, M. Ueda, Y. Miura, K. Sato, S. Bosu, K. Saito, M. Shirai, T.J. Konno, K.Takanashi, Extensive study of giant magnetoresistance properties in half-metallic $\mathrm{Co}_{2}(\mathrm{Fe}, \mathrm{Mn})$ Si-based devices, Applied Physics Letters, 101 (2012) 252408.

[10] I. Galanakis, P.H. Dederichs, N. Papanikolaou, Slater-Pauling behavior and origin of the half-metallicity of the full-Heusler alloys, Physical Review B, 66 (2002) 174429.

[11] T. Ishikawa, T. Marukame, H. Kijima, K.I. Matsuda, T. Uemura, M. Arita, M. Yamamoto, Spin-dependent tunneling characteristics of fully epitaxial magnetic tunneling junctions with a full-Heusler alloy $\mathrm{Co}_{2} \mathrm{MnSi}$ thin film and a $\mathrm{MgO}$ tunnel barrier, Applied Physics Letters, 89 (2006) 192505.

[12] N. Tezuka, N. Ikeda, S. Sugimoto, K. Inomata, Giant tunnel magnetoresistance at room temperature for junctions using Full-Heusler $\mathrm{Co}_{2} \mathrm{FeAl}_{0.5} \mathrm{Si}_{0.5}$ electrodes, Japanese Journal of Applied Physics, 46 (2007) L454-L456.

[13] W. Wang, H. Sukegawa, K. Inomata, Temperature dependence of tunneling magnetoresistance in epitaxial magnetic tunnel junctions using a $\mathrm{Co}_{2} \mathrm{FeAl}$ Heusler alloy electrode, Physical Review B, 82 (2010) 092402.

[14] M.I. Katsnelson, V.Y. Irkhin, L. Chioncel, A.I. Lichtenstein, R.A. de Groot, Half-metallic ferromagnets: From band structure to many-body effects, Reviews of Modern Physics, 80 (2008) 315-378.

[15] I. Galanakis, Surface properties of the half-and full-Heusler alloys, Journal of Physics: Condensed Matter, 14 (2002) 6329.

[16] H.J. Elmers, S. Wurmehl, G.H. Fecher, G. Jakob, C. Felser, G. Schönhense, Field dependence of orbital magnetic moments in the Heusler compounds $\mathrm{Co}_{2} \mathrm{Fe} \mathrm{Al}$ and $\mathrm{Co}_{2} \mathrm{Cr}_{0.6} \mathrm{Fe}_{0.4} \mathrm{Al}$, Applied Physics A, 79 (2004) 557-563.

[17] N.I. Kourov, V.V. Marchenkov, Y.A. Perevozchikova, H.W. Weber, The role of specific features of the electronic structure in electrical resistivity of band ferromagnets $\mathrm{Co}_{2} \mathrm{FeZ}(\mathrm{Z}$ = Al, Si, Ga, Ge, In, Sn, Sb), Physics of the Solid State, 59 (2017) 898-903.

[18] S. Sun, C.B. Murray, D. Weller, L. Folks, A. Moser, Monodisperse FePt nanoparticles and ferromagnetic FePt nanocrystal superlattices, science, 287 (2000) 1989-1992. 
[19] S. Sun, Recent advances in chemical synthesis, self-assembly, and applications of FePt nanoparticles, Advanced Materials, 18 (2006) 393-403.

[20] J.H. Lee, J.W. Kim, J. Cheon, Magnetic nanoparticles for multi-imaging and drug delivery, Molecules and cells, 35 (2013) 274-284.

[21] Z. Wang, X. Wang, M. Li, Y. Gao, Z. Hu, T. Nan, X. Liang, H. Chen, J. Yang, S. Cash, N.X. Sun, Highly sensitive flexible magnetic sensor based on anisotropic magnetoresistance effect, Adv Mater, 28 (2016) 9370-9377.

[22] Z. Hu, B. Ma, R.E. Koritala, U. Balachandran, Temperature-dependent energy storage properties of antiferroelectric $\mathrm{Pb}_{0.96} \mathrm{La}_{0.04} \mathrm{Zr}_{0.98} \mathrm{Ti}_{0.02} \mathrm{O} 3$ thin films, Applied Physics Letters, 104 (2014) 263902.

[23] Z. Wang, Y. Zhang, Y. Wang, Y. Li, H. Luo, J. Li, D. Viehland, Magnetoelectric assisted 180 magnetization switching for electric field addressable writing in magnetoresistive random-access memory, ACS nano, 8 (2014) 7793-7800.

[24] F. Yang, D. Liu, W. Li, P. Xiong, Y. Jia, X. Chen, C. Yang, Morphology, microstructure and magnetic properties of $\mathrm{Co}_{2} \mathrm{FeAl}$ alloy nanostructures prepared at low temperature, Journal of Alloys and Compounds, 735 (2018) 1206-1210.

[25] K.R. Sapkota, P. Gyawali, A. Forbes, I.L. Pegg, J. Philip, Synthesis and characterization of $\mathrm{Co}_{2} \mathrm{FeAl}$ nanowires, Journal of Applied Physics, 111 (2012) 123906.

[26] M. Almasi-Kashi, A. Ramazani, S. Alikhanzadeh-Arani, Z. Pezeshki-Nejad, A. Hassan Montazer, Synthesis, characterization and magnetic properties of hollow $\mathrm{Co}_{2} \mathrm{Fe} \mathrm{Al}$ nanoparticles: the effects of heating rate, New Journal of Chemistry, 40 (2016) 5061-5070.

[27] H. Al-Kanani, J. Booth, Magnetic and structural studies of Fe and Ni substituted CoAl alloys, Journal of magnetism and magnetic materials, 139 (1995) 299-311.

[28] A. Kumar, P.C. Srivastava, Synthesis and characterization of $\mathrm{Co}_{2} \mathrm{FeAl}$ Heusler alloy nanoparticles, Materials Science-Poland, 31 (2013) 501-505.

[29] J. Du, Y. Zuo, Z. Wang, J. Ma, L. Xi, Properties of $\mathrm{Co}_{2} \mathrm{FeAl}$ Heusler alloy nano-particles synthesized by coprecipitation and thermal deoxidization method, Journal of Materials Science \& Technology, 29 (2013) 245-248.

[30] L.T. Huynh, S.N. Bonvicini, A.C. Pinon, S. Trudel, Nanocrystalline alloys: synthesis and characterization of non-stoichiometric $\mathrm{Co}_{2} \mathrm{FeAl}$ nanocrystals, Canadian Journal of Chemistry, 94 (2015) 367-372.

[31] K. Inomata, N. Ikeda, N. Tezuka, R. Goto, S. Sugimoto, M. Wojcik, E. Jedryka, Highly spin-polarized materials and devices for spintronics, Science and technology of advanced materials, 9 (2008) 014101.

[32] K. Kobayashi, R.Y. Umetsu, R. Kainuma, K. Ishida, T. Oyamada, A. Fujita, K. Fukamichi, Phase separation and magnetic properties of half-metal-type $\mathrm{Co}_{2} \mathrm{Cr}_{1-\mathrm{x}} \mathrm{Fe}_{\mathrm{x}} \mathrm{Al}$ alloys, Applied Physics Letters, 85 (2004) 4684-4686.

[33] C. Wang, A.A. Levin, J. Karel, S. Fabbrici, J. Qian, C.E. ViolBarbosa, S. Ouardi, F. Albertini, W. Schnelle, J. Rohlicek, G.H. Fecher, C. Felser, Size-dependent structural and magnetic properties of chemically synthesized Co-Ni-Ga nanoparticles, Nano Research, 10 (2017) 3421-3433.

[34] S. Wurmehl, G.H. Fecher, H.C. Kandpal, V. Ksenofontov, C. Felser, H.-J. Lin, Investigation of $\mathrm{Co}_{2} \mathrm{FeSi}$ : The Heusler compound with highest Curie temperature and magnetic moment, Applied Physics Letters, 88 (2006) 032503. 
[35] A. Aslani, M. Ghahremani, M. Zhang, L.H. Bennett, E. Della Torre, Enhanced magnetic properties of $\mathrm{Ni}_{51} \mathrm{Mn}_{33.4} \mathrm{In}_{15.6}$ Heusler alloy nanoparticles, IEEE Transactions on Magnetics, 53 (2017) 1-6.

[36] A. Aslani, M. Ghahremani, M. Zhang, L.H. Bennett, E. Della Torre, Customizing magnetic and structural properties of nanomaterials, IEEE Transactions on Magnetics, 54 (2018) 1-5. 